\title{
Electromagnetic pulse reflection at self-generated plasma mirrors: laser pulse shaping and high order harmonic generation.
}

\author{
S. S. Bulanov, ${ }^{1,2}$ A. Macchi, ${ }^{3}$ A. Maksimchuk, ${ }^{1}$ T. Matsuoka, ${ }^{1}$ J. Nees,${ }^{1}$ and F. Pegoraro ${ }^{4}$ \\ ${ }^{1}$ FOCUS center and Center for Ultrafast Optical Science, \\ University of Michigan, University of Michigan, Ann Arbor, USA \\ ${ }^{2}$ Institute of Theoretical and Experimental Physics, Moscow, Russia \\ ${ }^{3}$ polyLAB, CNR-INFM, University of Pisa, Pisa, Italy \\ ${ }^{4}$ Department of Physics, University of Pisa and CNISM, Pisa, Italy
}

\begin{abstract}
A thin layer of overdense plasma is created when an electromagnetic pulse interacts with a rapidly ionizing thin foil. This layer will reflect the incoming pulse, forming a so-called plasma mirror. A simple realistic model based on paired kinetic and wave equations is used to describe analytically the process of mirror formation and the reflection and transmission of the incident pulse. The model incorporates the exact description of the ionization process in the foil and the polarization and conduction currents that follow. The analytical description of the reflected and transmitted pulses as well as their dependence on foil parameters, and initial pulse amplitude and form are presented. Possible application and effectiveness of this process to improve laser pulse contrast are discussed. In the case of the linearly polarized incident pulse, there harmonic generation occurs, that is absent in the case of the circular polarization. The spectra of the reflected pulses for different initial forms and amplitudes are studied.
\end{abstract}

PACS numbers: 52.40.Hf, 52.50.Jm, 42.65.Ky

Keywords: Ionization, plasma mirror, wave propagation in plasma, high order harmonics

\section{INTRODUCTION}

The interaction of ultrashort, high-intensity laser pulses with matter has lead to a new regime of nonlinear optics where the optical properties of the medium undergo ultrafast changes due to ionization produced by a laser pulse. In this context, by ultrashort, high-intensity pulses we refer to pulses with typical duration in the sub-picosecond regime, and whose intensity is high enough to cause field ionization of matter within a very few optical cycles, or even a sub-cycle interval. Therefore, by ultrafast changes of, e.g., a change in the index of refraction we mean that a latter varies significantly over a laser cycle, due to the rapid increase of the free electron density due to field ionization. As a consequence the pulse properties, such as envelope shape, spectrum and duration, can be strongly modified.

A number of papers have addressed the problem of modeling the pulse propagation in a fast ionizing medium (see e.g. [1]) and studied the possible applications of ionization effects for pulse shaping and manipulation. The case of a high-density material, such as a solid target, is of particular interest since the free electron density produced by ionization can become quickly high enough to make the plasma refractive index turn from real to imaginary values, leading to self-reflection [2, 3]. This effect is the physical basis for the so-called "plasma mirror" where plasma production at the surface of a mirror is used experimentally to obtain "clean" ultrashort pulses by removing the leading edge of the low-intensity "prepulse" or "pedestal", which characterizes femtosecond laser systems [4, [5, 6, 7], by letting it be trasmitted through the mirror before the ionization-induced transition to opacity occurs. The resulting improvement of the laser pulse contrast (defined as the ratio between the prepulse energy and the short pulse energy) is important for various applications of current importance. These latter include, for example, the acceleration of fast ions from solid targets (see [8] and references therein) where avoiding plasma formation at the target surface by the prepulse may enhance the ion source efficiency [9]. Similarly, high-contrast pulse may optimize high harmonic generation from solid target surfaces [10] Another proposed application is the use of the combined effects of ultrafast optical shuttering and harmonic generation to obtain a sub-femtosecond pulse in transmission [11].

In these application regimes a thin (i.e., having a thickness less than an optical wavelength) foil target may be used in principle because the thickness of the reflecting plasma layer is usually of the order of the plasma skin depth. On the other hand the foil thickness may be used as an additional control parameter for applications. From the point of view of theoretical modeling a thin foil target is of interest because it allows an analytical treatment to some larger extent (see e.g. 11, 12]), thus providing an useful reference case for models and numerical simulations, and possibly aiding further optimization of the plasma mirror functionality. As a further reason of general interest, the laserplasma interaction dynamics in a thin foil includes peculiar features that are not encountered either in underdense or overdense plasmas [13, 14].

In the present paper we utilize a simple analytic model for a dielectric foil ionization by an electromagnetic pulse. In order to describe the circularly polarized pulse interaction with the foil we use a kinetic equation paired with a wave equation. The effects of ionization are incorporated into the source term in the kinetic equation in the form 
of a differential ionization rate [15, 16]. The solution of the kinetic equation, the momentum distribution function, enters the expression for the conduction and polarization currents that emerge in the foil. We point out differing behaviors between conduction and polarization electric currents. While the first grows with the increase of charged particle number, the second is proportional to the ionization rate, which means that it will be most important in the beginning of the ionization. In order to describe the field evolution during the interaction with the foil we utilize the wave equation with nonlinear boundary conditions, obtained in Ref. [12]. We show that as the pulse ionizes the foil its reflectivity grows giving rise to the reflected pulse. The form of the reflected and transmitted pulses depends strongly on the initial amplitude of the pulse. We study the process of plasma mirror formation under the action of a pulse with a gaussian envelope that has a pedestal. There is a significant pedestal reduction after the pulse reflection from the ionized foil. However there is a dependence of the reduction efficiency on the initial contrast of the pulse, which we study. We also consider the interaction of a linearly polarized pulse with a thin dielectric foil. In this case high order harmonics are generated due to the nonlinear dependence of the ionization rate on the pulse amplitude. We study the high order harmonic generation efficiency dependence on the initial amplitude of the pulse and on the form of the pulse.

The paper is organized as follows. In section 2 we review the solution of the Maxwell equations with nonlinear boundary conditions. In section 3 we carry out the kinetic description of the currents that enter the wave equation. In section 4 we consider an analytical solution of the simplified wave equation. The results of the numerical solution in the case of a circularly polarized initial pulse are presented in section 5. In section 6 a linearly polarized pulse interaction with a foil is studied numerically. We summarize conclusions in section 7 .

\section{WAVE EQUATION WITH NONLINEAR BOUNDARY CONDITIONS}

In this section we review the solution of the wave equation with nonlinear boundary conditions as it was presented in Ref. [12]. We consider the case of a circularly polarized plane wave impinging at normal incidence on a hydrogen-like foil, which is located in the plane $x=0$. The interaction of the pulse with the foil is taken to be uniform along the foil surface, which is justified by the fact that plasma mirrors operate in the regime in which the laser pulse is weakly focused on a mirror with a spot size of hundreds of wavelengths [4, [5, 6, 7]. Relativistic effects of the ionization of the foil are neglected, which is valid if the magnetic field contribution to the ionization probability as well as to the electron motion is small. The density of the foil is taken to be high enough to ensure that the plasma density is not restrained by the fact that the number of neutral atoms might be exhausted. Also it is assumed that the electrons that emerge in the foil during the ionization remain localized within the foil due to the charge separation field produced in the presence of immobile heavy ions. So, the current is proportional to $\delta(x)$, where $\delta(x)$ is Dirac delta function. The wave equation for the vector potential $\mathbf{A}(x, t)$ is

$$
\partial_{t t} \mathbf{A}-c^{2} \partial_{x x} \mathbf{A}=4 \pi c \delta(x) \mathbf{J}(\mathbf{A})+\delta^{\prime}(t) \mathbf{A}(x, 0)+\delta(t) \dot{\mathbf{A}}(x, 0) .
$$

The first term on the RHS of Eq. (11) describes the electric current, localized in the foil, which is a functional of the vector potential taken at $x=0$. The last two terms are equivalent to the initial conditions $\mathbf{A}(x, 0)=\mathbf{A}_{0}(x)$, $\partial_{t} \mathbf{A}(x, 0)=\dot{\mathbf{A}}_{0}(x)$, where $\mathbf{A}_{0}(x)$ and $\dot{\mathbf{A}}_{0}(x)$ define the incident electromagnetic pulse.

The solution of Eq. (11) can be presented as [12]:

$$
\mathbf{A}(x, t)=\mathbf{A}_{0}(x, t)+2 \pi \int_{0}^{t-|x| / c} \mathbf{J}(\mathbf{A}(0, \tau)) d \tau
$$

By differentiating this equation with respect to time or spatial coordinate $x$ we obtain the explicit form of the electric or magnetic field respectively as functions of the incident electric field $\mathbf{E}_{0}(x, t)$ and $\mathbf{A}(0, t)$, which can be found from Eq. (2) taken at $x=0$.

$$
\begin{gathered}
\mathbf{E}(x, t)=\mathbf{E}_{0}(x, t)-\frac{2 \pi}{c} \mathbf{J}(\mathbf{A}(0, t-|x| / c)), \\
\mathbf{B}(x, t)=\mathbf{B}_{0}(x, t)+\frac{2 \pi}{c} \mathbf{e}_{x} \times \mathbf{J}(\mathbf{A}(0, t-|x| / c)) \operatorname{sign}(x),
\end{gathered}
$$

where $\mathbf{e}_{x}$ is the unit vector along the direction of wave propagation. In order to solve these equations one should determine the expression for the current. 


\section{KINETIC DESCRIPTION OF THE CURRENT}

The behavior of the electrons in the foil in the presence of the homogeneous time-varying electric field can be described by the kinetic equation of the following form

$$
\frac{\partial f(\mathbf{p}, t)}{\partial t}-e \mathbf{E}(t) \frac{\partial f(\mathbf{p}, t)}{\partial \mathbf{p}}=q(\mathbf{p}, \mathbf{E}, t)
$$

where the distribution function $f(\mathbf{p}, t)$ is normalized in such a way that $\int f(\mathbf{p}, t) d^{3} p$ gives the number of electrons per unit volume. (Similar approaches to field ionization modeling can be found, e.g., in Ref. [17, 18]). Since we are considering the nonrelativistic regime, we can neglect the effects of the incident magnetic field, which are suppressed by the factor $v / c$, in the motion of electrons as well as in the ionization. The source term, $q(\mathbf{p}, \mathbf{E}, t)$, is connected with ionization of the foil by the incident electromagnetic pulse in such a way that $\int q(\mathbf{p}, \mathbf{E}, t) d^{3} p$ gives the total ionization probability $W=8 n_{a} \omega K_{0} \kappa^{3} \mathcal{E}^{-1} \exp \left[-2 \kappa^{3} / 3 \mathcal{E}\right]$ of the unit volume in unit time (the time is measured in units of $\left.\hbar^{3} / m e^{4}\right)$. It is determined by the differential probability of ionization by a circularly polarized electric field [16]

$$
q=\frac{4 \kappa^{4}}{\pi^{2}} \frac{n_{a} \omega K_{0}}{\mathcal{E}^{2}} \exp \left[-\frac{2 \kappa^{3}}{3 \mathcal{E}}\right] \exp \left\{-\frac{\kappa}{\mathcal{E}}\left[\left(\frac{p_{\perp}}{m c}-\frac{\mathcal{E}}{\omega} \frac{(4 \pi \alpha)^{2} c}{a_{B}}\right)^{2}+\left(\frac{p_{x}}{m c}\right)^{2}\right]\right\},
$$

where $\omega$ is the frequency of the incident electric field, $\mathcal{E}=E / E_{a}$ is the normalized amplitude of the electric field, $E_{a}=m^{2} e^{5} / \hbar^{4}=5.14 \cdot 10^{9} \mathrm{~V} / \mathrm{cm}$ is characteristic atomic field, $\kappa=\sqrt{I / I_{H}}, I$ is the ionization potential and $I_{H}=m e^{4} / 2 \hbar^{2}=13.6 \mathrm{eV}$ is the ionization potential of the hydrogen atom, $K_{0}=I / \hbar \omega, n_{a}$ is the density of neutral atoms, $a_{B}=\hbar^{2} / m e^{2}$ is Bohr radius, and $\alpha=1 / 137$ is fine-structure constant. The component of electron momentum perpendicular to the direction of pulse propagation is denoted by $p_{\perp}$, and along the direction of pulse propagation is $p_{x}$. The momentum distribution of produced electrons is very narrow with maximum at $p_{x}=0$, $p_{\perp} / m c=(\mathcal{E} / \omega)\left(16 \pi^{2} \alpha c / a_{B}\right)$ [19, 20]. This means that electrons preferably emerge from under the barrier with momentum perpendicular to the instantaneous direction of the electric field and equal to that of a free electron acquires in such a field. Since the momentum distribution is very narrow, we can use delta functions instead of exponents in order to simplify the calculations:

$$
q=\frac{8 \kappa^{3} K_{0} n_{a} \omega}{\mathcal{E}} \exp \left[-\frac{2 \kappa^{3}}{3 \mathcal{E}}\right] \delta\left(\frac{p_{\perp}}{m c}-\frac{\mathcal{E}}{\omega} \frac{(4 \pi \alpha)^{2} c}{a_{B}}\right) \delta\left(p_{x}\right)
$$

We should also note that in the expression for the source term the absolute value of the field enters.

We solve Eq.(5) by integrating it along the particle characteristics. The equations for the characteristics are

$$
p_{0}=p_{0}(0), \quad \frac{d p_{\perp}}{d t}=-e E, \quad \frac{d f}{d t}=q .
$$

Introducing the function $\mathbf{A}(t)=-\int_{0}^{t} \mathbf{E} d s$, we obtain

$$
\mathbf{p}_{\perp}=-e \int_{0}^{t} \mathbf{E} d s+\mathbf{p}_{\perp 0} \quad \text { i.e., } \quad \mathbf{p}_{\perp}-e \mathbf{A}(t)=\mathbf{p}_{\perp 0} .
$$

As a result the distribution function is given by the following functional

$$
f=f_{0}\left[p_{x}, \mathbf{p}_{\perp}-e \mathbf{A}(t)\right]+\int_{0}^{t} q\left\{\mathbf{p}_{\perp}-e\left[\mathbf{A}(t)-\mathbf{A}\left(t^{\prime}\right)\right], t^{\prime}\right\} d t^{\prime},
$$

where $f_{0}\left(p_{x}, \mathbf{p}_{\perp}\right)$ is the distribution function of the initial electrons before the passage of the electromagnetic wave. In our case $f_{0}=0$, since the foil was not ionized initially. The appearance of electrons due to the ionization of the foil gives rise to conduction and polarization electric currents. The conduction electric current is due to the electron motion in the field of the incident pulse, while the polarization electric current is due to the ionization process, since when the neutral atom is ionized an electric dipole is created. 
In order to determine the form of the polarization electric current we write down the second moment of the kinetic equation

$$
\begin{array}{r}
\sum_{\alpha} \int\left[\left(m_{\alpha}^{2} c^{4}+p^{2} c^{2}\right)^{1 / 2}-m_{\alpha} c^{2}\right]\left\{\frac{\partial f_{\alpha}(\mathbf{p}, t)}{\partial t}+e_{\alpha} \mathbf{E}(t) \frac{\partial f_{\alpha}(\mathbf{p}, t)}{\partial \mathbf{p}}\right\} d^{3} p \\
=\sum_{\alpha} \int\left[\left(m_{\alpha}^{2} c^{4}+p^{2} c^{2}\right)^{1 / 2}-m_{\alpha} c^{2}\right] q_{\alpha}(\mathbf{E}, \mathbf{p}, t) d^{3} p
\end{array}
$$

which we rewrite as

$$
\frac{\partial K}{\partial t}-\mathbf{j}_{\text {cond }} \mathbf{E}=\Sigma-\sum_{\alpha} m_{\alpha} \frac{\partial n_{\alpha}}{\partial t}, \quad \mathbf{j}_{\text {cond }}=e \int f \frac{\mathbf{p}_{\perp} c}{\sqrt{m^{2} c^{4}+\mathbf{p}^{2} c^{2}}} d^{3} p
$$

where $K=\sum_{\alpha} \int\left[\left(m_{\alpha}^{2} c^{4}+p^{2} c^{2}\right)^{1 / 2}-m_{\alpha} c^{2}\right] f_{\alpha} d^{3} p$ is the kinetic energy and $\Sigma=\sum_{\alpha} \int\left(m_{\alpha}^{2} c^{4}+p^{2} c^{2}\right)^{1 / 2} q_{\alpha}(\mathbf{E}, \mathbf{p}, t) d^{3} p$. Since in the foil not only electrons and ions are present but also neutral atoms, from which by the process of ionization the plasma is created, $\partial n_{e} / \partial t=\partial n_{i} / \partial t=-\partial n_{a} / \partial t$. Here indexes $e, i$, and $a$ stand for electron, ion, and atom respectively. Then

$$
\sum_{\alpha} m_{\alpha} c^{2} \frac{\partial n_{\alpha}}{\partial t}=\left(m_{e}+m_{i}-m_{a}\right) c^{2} \frac{\partial n_{e}}{\partial t}=I \frac{\partial n_{e}}{\partial t}
$$

where $I=\left(m_{e}+m_{i}-m_{a}\right) c^{2}$, and is usually referred to as a mass defect or ionization potential, which is due to the fact that some electrons and ions are bound in atoms. In order to ionize an atom some amount of energy should be spent. So we rewrite Eq. (12) in the following form

$$
\frac{\partial}{\partial t}\left(K+I n_{e}\right)=\mathbf{j}_{\text {cond }} \mathbf{E}+\Sigma \text {. }
$$

The energy balance equation for the circularly polarized electromagnetic wave is

$$
\frac{\partial}{\partial t}\left(\frac{\mathbf{E}^{2}}{4 \pi}\right)=-\mathbf{j}_{\text {cond }} \mathbf{E}-\mathbf{j}_{p o l} \mathbf{E}
$$

Here we have taken into account that no external electric current is present in our case. Adding these two equations we obtain the energy balance equation for the system of electromagnetic wave and particles

$$
\frac{\partial}{\partial t}\left(\frac{\mathbf{E}^{2}}{4 \pi}+K+I n_{e}\right)=-\mathbf{j}_{p o l} \mathbf{E}+\Sigma .
$$

The RHS of this equation should be equal to zero, because of the energy conservation law. Since we are considering a nonrelativistic case, we can neglect $p_{\alpha}$ with respect to $m_{\alpha}$. Then the expression for polarization current will take the following form

$$
\mathbf{j}_{p o l}=\frac{\mathbf{E}(t)}{|\mathbf{E}(t)|^{2}} I \frac{\partial n_{e}}{\partial t} .
$$

To our knowledge the polarization current was first introduced in Ref. 21] while an extended discussion is given in Ref.[17]. The expression given in Ref.[17] is found to be identical to our formula (17) when one realizes that the "ejection energy" of freed electrons $\mathcal{E}_{I}$ has been included in $I$. For our aims one may assume $\mathcal{E}_{I}=0$, i.e. we assume free electrons appear with zero energy, as an acceptable simplifying assumption; in such a case $I$ represents in practice the standard ionization potential. In the more general case $I$ becomes a smooth function of the electric field.

It can be seen from Eq. (17) that the polarization electric current is along the direction of the electric field and is proportional to the ionization potential and to the ionization rate. Due to this fact we expect the contribution of the polarization electric current to be rather small. However, if we consider the ionization of heavy multicharged ions (where the binding energy of low lying levels is comparable to the electron rest mass) by an intense electromagnetic pulse, the polarization electric current can play an important role. It is similar to the case of electron-positron pair production by intense electromagnetic wave in plasma [22], where the polarization electric current is proportional to $2 m_{e}$. In that case the polarization electric current totally changes the behavior of the wave, forcing the vector potential to oscillate around some nonzero value. Also the components of the electric field begin to oscillate with different frequencies. So in the process where the backreaction of the produced particles on the properties of the wave 
that created them takes place, the polarization electric current should be taken into account. In the case of a very sharp electromagnetic pulse front, consider, for example, the interaction of high contrast and very intense pulse with the foil, the ionization rate is very high, and the polarization electric current will be significant, since the conduction electric current will not be able to develop large value due to the absence of electrons and ions in the first moments of evolution.

Using the explicit expression for the distribution function (10) and the source term (77), and substituting (12 17) into Eq. (3), we obtain:

$$
\begin{array}{r}
\mathbf{E}(x, t)=\mathbf{E}_{0}(x, t)-\frac{2 \pi}{c} \frac{e^{2} l}{m} \mathbf{A}(0, t-|x| / c) \int_{0}^{t-|x| / c} \frac{8 \kappa^{3} K_{0} n_{a} \omega}{\mathcal{E}} \exp \left[-\frac{2 \kappa^{3}}{3 \mathcal{E}}\right] d t^{\prime} \\
-\frac{2 \pi}{c} \frac{\mathbf{E}(0, t-|x| / c)}{|\mathbf{E}(0, t-|x| / c)|^{2}} \frac{8 I l \kappa^{3} K_{0} n_{a} \omega}{\mathcal{E}} \exp \left[-\frac{2 \kappa^{3}}{3 \mathcal{E}}\right] .
\end{array}
$$

We can rewrite the equation for the electric field in terms of dimensionless field and vector potential, $\eta=e \mathbf{E} / m c \omega_{0}$, $\mathbf{a}=e \mathbf{A} / m c^{2}$, where $\omega_{0}$ is the frequency of the incident wave. The density of the plasma, that emerges due to the ionization, is taken in the units of $n_{0}=m \omega_{p e}^{2} / 4 \pi e^{2}$, the critical plasma density, corresponding to the frequency of the initial pulse with time in the units of inverse frequency, $\omega_{0}$. The equation that governs the behavior of the dimensionless electric field is

$$
\eta(x, t)=\eta_{0}(x, t)-\epsilon_{p} \mathbf{a}(0, t-|x / c|) n(0, t-|x / c|)-\epsilon_{p} \frac{I}{m c^{2}} \frac{\eta(0, t-|x / c|)}{|\eta(0, t-|x / c|)|^{2}} \dot{n}(0, t-|x / c|),
$$

where

$$
n(t)=\frac{n_{a}}{n_{0}} \int_{0}^{t} \frac{8 \kappa^{3} K_{0} \eta_{a}}{\left|\eta\left(t^{\prime}\right)\right|} \exp \left[-\frac{2 \kappa^{3} \eta_{a}}{3\left|\eta\left(t^{\prime}\right)\right|}\right] d t^{\prime} \quad \text { and } \quad \dot{n}(t)=\frac{n_{a}}{n_{0}} \frac{8 \kappa^{3} K_{0} \eta_{a}}{|\eta(t)|} \exp \left[-\frac{2 \kappa^{3} \eta_{a}}{3|\eta(t)|}\right]
$$

The normalized atomic field is denoted as $\eta_{a}=e E_{a} / m \omega c$ and the normalized density of the foil, $\epsilon_{p}=\omega_{p e}^{2} l / 2 \omega_{0} c$, is a crucial parameter of the laser pulse interaction with a thin plasma layer introduced in Ref. [13. It plays an important role not only in the process of electromagnetic pulse reflection/transmission at the thin foil [12], but also in the process of ion acceleration [13], when an intense laser pulse interacts with the thin foil, as well as in the process of high order harmonic generation in the relativistic regime of laser-foil interaction [23].

Equation (19) demonstrates that the conduction electric current is proportional to the number of electrons (ions), i.e. to plasma density, while the polarization electric current is proportional to the ionization rate, which makes it especially important in the first moments of intense ionization, should it occur. This distinction is due to the fact that the conduction electric current is connected with the motion of charged particles, while the polarization electric current is due to dipole creation through the ionization of neutral atoms.

Due to the nonlinear dependence of the current on the electric field amplitude the above equation is difficult to solve analytically.

\section{MODEL CASE}

In order to study the properties of Eq. (19) we consider a simplified model case. Let us assume that $n(t)$ and thus $\dot{n}(t)$ are given functions of time. Then the equation (19), governing the evolution of the electric field, can be rewritten at the foil $(x=0)$ in the following form

$$
\eta=\eta_{0}-N \mathbf{a}+\frac{\eta}{|\eta|^{2}} N_{1}, \quad \text { where } N=\epsilon_{p} n(t) \text { and } \quad N_{1}=\epsilon_{p} \frac{I}{m c^{2}} \frac{\partial n(t)}{\partial t}
$$

The initial electromagnetic wave is

$$
\eta_{0}=\eta_{i n} e^{i t}
$$

We shall look for the solution of Eq. (21) in the following form

$$
\eta=\eta e^{i(t+\varphi)} .
$$


After substituting the initial field and the solution into Eq. (21) we obtain a system of algebraic equations for the amplitude and the phase of the transmitted wave:

$$
\begin{aligned}
& \left(\eta^{2}+N_{1}\right) \cos \varphi=\eta \eta_{i n}-\eta^{2} N \sin \varphi \\
& \left(\eta^{2}+N_{1}\right) \sin \varphi=\eta^{2} N \cos \varphi
\end{aligned}
$$

Using the second equation we get the following expression for $\varphi$ :

$$
\varphi=\arctan \frac{\eta^{2} N}{\eta^{2}+N_{1}}
$$

Substituting this expression into the first equation of the system (24), we find a forth order algebraic equation for $\eta$ :

$$
\eta^{4}\left(1+N^{2}\right)+\eta^{2}\left(2 N_{1}-\eta_{i n}\right)+N_{1}^{2}=0 .
$$

The solutions of this equation are

$$
\eta= \pm\left[\frac{-\left(2 N_{1}-\eta_{i n}^{2}\right)+\sqrt{\left(2 N_{1}-\eta_{i n}^{2}\right)^{2}-4\left(1+N^{2}\right) N_{1}^{2}}}{2\left(1+N^{2}\right)}\right]^{1 / 2} .
$$

Two other roots of forth order equation were omitted since $\eta$ should be real.

Let us consider the moment of time, when there is no longer any ionization, because all the atoms that could be ionized by the pulse have been ionized. The plasma density reached its maximum and the main part of the incoming radiation is reflected by plasma mirror. In this case $N_{1}$ has $\partial n(t) / \partial t=0$, and

$$
\eta= \pm \frac{\eta_{i n}}{\sqrt{1+N^{2}}}
$$

This result states that even for plasma density much greater than critical there is a transmission of the incoming pulse through the ionized foil. However this transmission is suppressed by increased plasma density.

Let us investigate the phase behavior. Using Eq. (25), we find

$$
\varphi=\arctan \frac{\eta^{2} N}{\eta^{2}+N_{1}} \approx \arctan N
$$

The phase variation with time is

$$
\frac{\partial \varphi}{\partial t}=\frac{\dot{N}}{1+N^{2}}>0
$$

So there is a blue shift in transmitted pulse frequency, while the density of plasma increases (see also [24]). However in the region with $N=$ const and $N_{1}=0$ there is no frequency shift and the radiation is transmitted without any frequency change. This fact should lead to the steepening of the transmitted pulse front and the rear should be of the same (half)width as the initial pulse. This can be seen from the results of numerical calculations, presented in the next section of this paper. So here unlike the case of the wave propagation in the ionized medium, the blue shift is in effect only when the ionization occurs. The frequency up-shift is connected with the rate of plasma density increase.

\section{THE RESULTS OF NUMERICAL SOLUTION}

In this section we present the results of numerical solution of Eq. (19) at the foil, $x=0$. We consider two cases of initial pulse form. First, the case of the pulse with gaussian envelope and gaussian pedestal, the maxima of the pulse and the pedestal temporaly coincide,

$$
E(0, t)=E_{0}\left\{p \exp \left[-\left(\frac{t-\tau / 2}{\tau / 2}\right)^{2}\right]+(1-p) \exp \left[-\left(\frac{t-\tau / 2}{\tau / 20}\right)^{2}\right]\right\} e^{i \omega_{0} t},
$$

where $p$ is the ratio of pedestal amplitude to the main pulse amplitude and $\tau=40 T$ is the pulse duration, $T=2 \pi / \omega_{0}$. Second, the case of

$$
E(0, t)=E_{0}[\sin (\pi t / \tau)]^{2} \exp \left[i \omega_{0} t\right],
$$



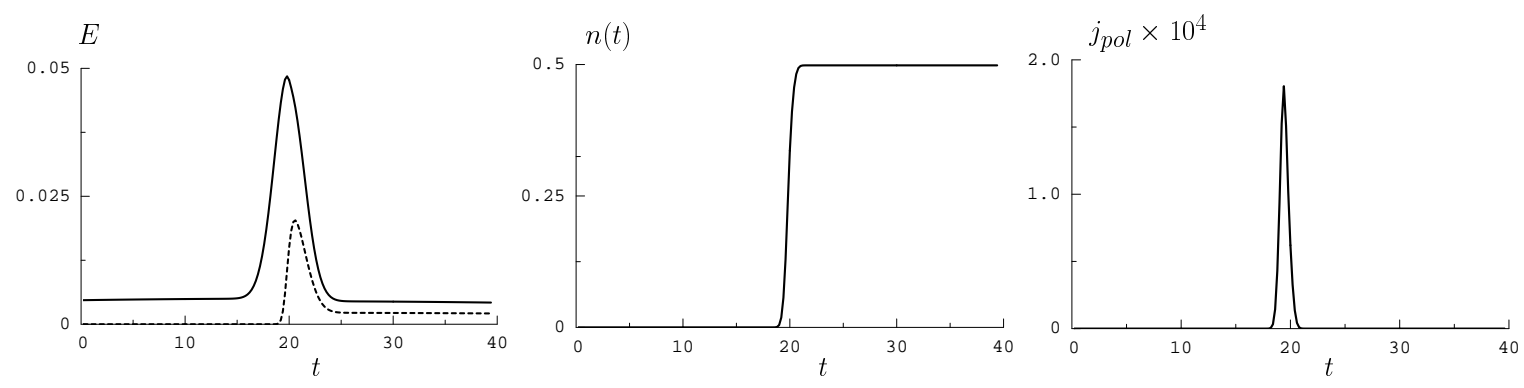

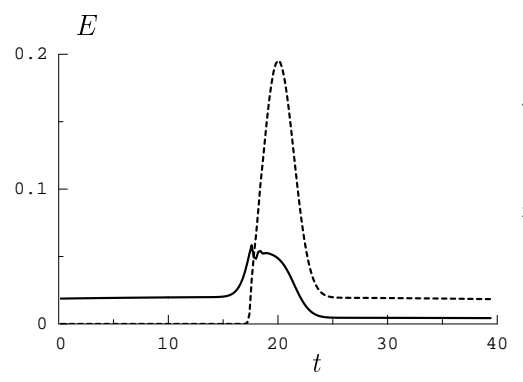

a)

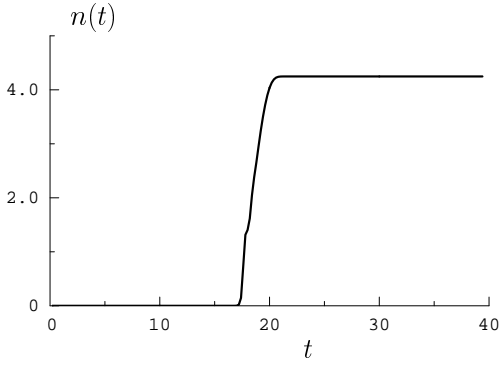

b)

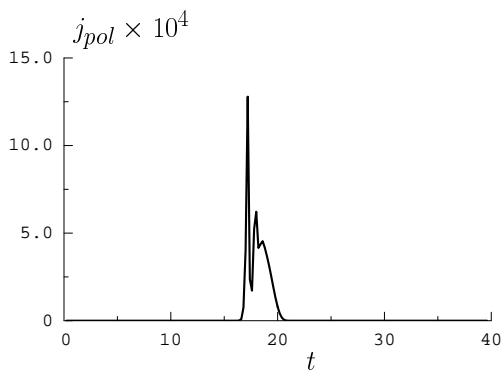

c)

FIG. 1: Evolution of the reflected (dotted curve) and transmitted pulse electric field (a), plasma density (b) and polarization current (c) for initial pulse of the form (31). Time is measured in units of wave period $(2 \pi / \omega)$, field is measured in units of $m c \omega / e$, density is measured in units of critical density, $n_{c r}$, and currents are dimensionless. The parameter $\epsilon_{p} K_{0}=4 \times 10^{3}$, $x=0$. The first row corresponds to the initial pulse amplitude equal to $\eta_{0}^{\max }=0.05$, the second row to $\eta_{0}^{\max }=0.2$.

We consider time interval $(0, \tau)$ to ensure that only one pulse is taken into account. This form of the pulse allows us to investigate the fronts of the reflected and transmitted pulses, since at $t=0$ the initial field is equal to zero.

In Figs. 1 and 2 we present the results of numerical solution of Eq. (19) with initial pulse having form (31) and (32) respectively. In Fig. 1 maximal electric field is $\eta_{0}^{\max }=0.05$ (first row) and $\eta_{0}^{\max }=0.2$ (second row). The amplitudes of the reflected and transmitted pulses are shown in Fig. 1a. The plasma density evolution which is closely connected with the conduction current is shown in Fig. 1b. The behavior of polarization current is presented in Fig. 1c. In the case of $\eta_{0}^{\max }=0.05$ (Fig. 1, first row) the incoming pulse is not intense enough to sufficiently ionize the foil, the resulting plasma density is 0.5 of critical. Due to this fact the reflectivity of the of the ionized foil is low, and the reflected pulse is much lower than the transmitted one. However it can clearly be seen that the pedestal is transmitted through the foil. The reflected pulse starts to differ from zero only when the main part of the initial pulse arrives at the foil.

In the case of $\eta_{0}^{\max }=0.2$ (Fig. 1, second row) the situation is different. Since the initial pulse intensity is high enough to produce a plasma with a density 4 times larger than critical by means of foil ionization, the reflectivity of the foil tends to unity, as shown in Fig. 1a the main pulse is almost completely reflected. However we should note that the intensity of the pedestal is not enough to ionize the foil, that is why it is completely transmitted through the foil. When the main pulse arrives at the foil it quickly ionizes the foil, it can be clearly seen in Fig 1b, second row, where the plasma density evolution is presented. In this case the reflected pulse is pedestal-free. So through the interaction of the intense electromagnetic pulse with rapidly ionized foil the reflected pulse is almost the same as the initial one apart from the fact that the pedestal is greatly reduced in the reflected pulse. We should also note that the reflected pulse has a steeper front than the initial one and its tail is of the same (half)width as the initial one (see Fig. 1a). These features are qualitatively expected from the analysis of the simplified wave equation carried out in the previous section.

In order to illustrate the contribution of the polarization current to the evolution of the reflected and transmitted pulses, we present Fig. 1c. As it was pointed out above, the expected polarization current contribution should be rather small, since it is proportional to the ionization potential. However the polarization current is also proportional to the ionization rate, so it can be important in the first moments of intense ionization. It can be seen from Fig. 1c that the polarization current is small compared to conduction one (to estimate the amplitude of the conduction current, one can multiply electric field amplitude by the number of particles), but it is non-zero in the region where the conduction current is far from its maximal value, and thus its contribution can be of the order of conduction current contribution.

In Fig. 2, where the results of numerical solution of Eq. (19) for initial pulse (32) are presented, maximal electric field is $\eta_{0}^{\max }=0.05$ (first row) and $\eta_{0}^{\max }=0.5$ (second row). The amplitudes of the reflected and transmitted pulses 

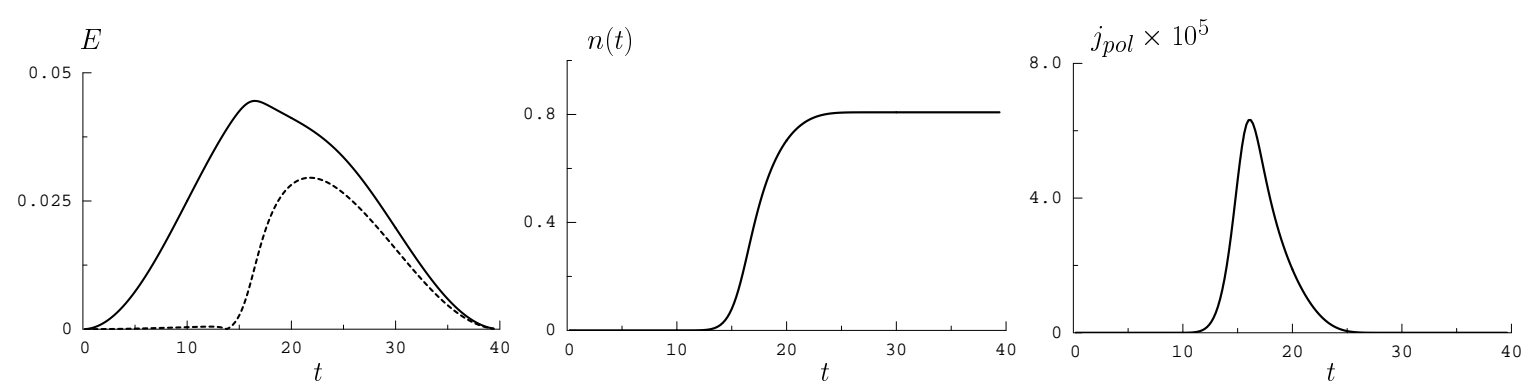

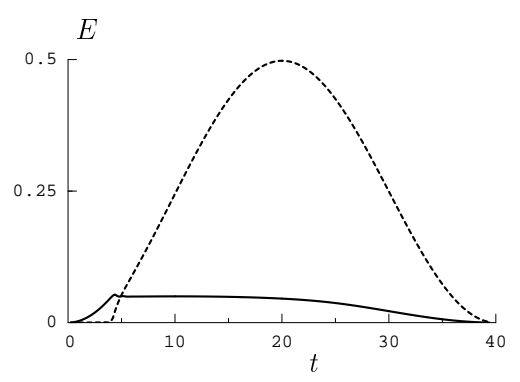

a)

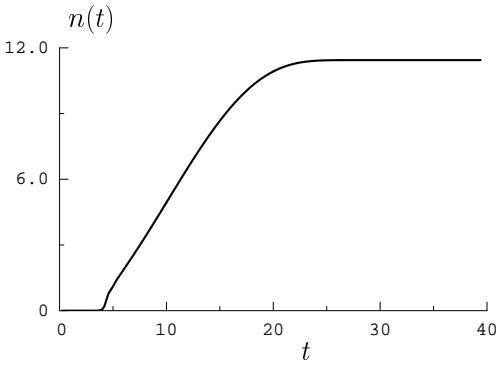

b)

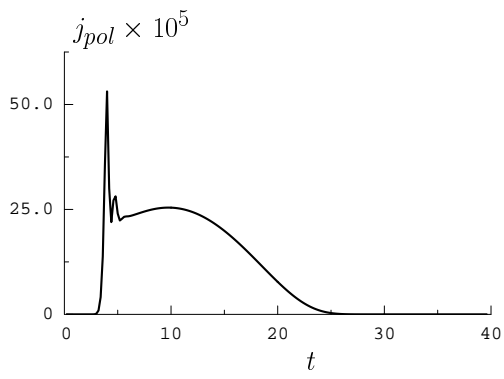

c)

FIG. 2: Evolution of the reflected (dotted curve) and transmitted pulse electric field (a), plasma density (b) and polarization current (c) for initial pulse of the form (32). Time is measured in units of wave period $(2 \pi / \omega)$, field is measured in units of $m c \omega / e$, density is measured in units of critical density, $n_{c r}$, and currents are dimensionless. The parameter $\epsilon_{p} K_{0}=4 \times 10^{3}$, $x=0$. The first row corresponds to an initial pulse amplitude equal to $\eta_{0}^{\max }=0.05$, the second row to $\eta_{0}^{\max }=0.5$.

are shown in Fig. 2a. The evolution of plasma density can be seen in Fig. 2b, and the behavior of polarization current in Fig. 2c. In the case of the initial pulse maximal amplitude of $\eta_{0}^{\max }=0.05$ (first row), the reflected and transmitted pulses are roughly of the same order, but the reflected pulse has a steeper front, since the reflectivity of the foil depends on the density of plasma, which evolution is shown in Fig. 2b. With increasing of the initial pulse amplitude the situation changes, in the case of $\eta_{0}^{\max }=0.5$ (second row) the transmitted pulse is much smaller than the reflected, which almost coincides with the initial one. Since the amplitude of the initial pulse is high enough to create a significant amount of plasma even in the first moments of the pulse interaction with the foil, the initial pulse gets reflected. Here, analogous to the case of the pulse with the pedestal in interaction with the foil, the reflected pulse has the characteristic temporal profile. Its front is steeper than its tail, while the latter (half)width is the same as the (half)width of the initial pulse. The properties of the polarization current are the same as in the previous case of pulse with pedestal. The absolute value of the polarization current is much smaller than that of the conduction current. However the polarization current has a maximum in the region where the conduction current is far from reaching its maximal value.

Let us consider the case of the pulse with pedestal once more but from the point of view of obtaining high contrast pulses. In order to get a high contrast pulse the pedestal should be transmitted through the foil and the safe-reflection should be triggered by the main pulse. In Fig. 3 we present a contour plot of the reflected pulse resulted contrast dependence on the initial pulse contrast and intensity. It is calculated as the ratio of the reflected pulse pedestal instantaneous intensity at the point where the pedestal and the main pulse envelopes intersect and the reflected pulse maximum intensity. The contours correspond to the values $10^{2 k}, k=1 . .10$ of the resulted contrast. We see that for pulses with the low initial contrast there is no way to improve it by the reflection on the rapidly ionized foil. It is due to the fact that the intensity of the pedestal is high enough to ionize the foil giving rise to the reflection of the incoming radiation. When the initial contrast improves there appears a region (to the right of the dashed line in Fig. 3) of initial pulse intensity values where the resulting contrast of the reflected pulse is better than that of the initial pulse. With the further improvement of initial pulse contrast this region enlarges, and for initial contrast better than $10^{-5}$, this region covers all the range of considered amplitudes of the initial pulse. So in order to improve the laser pulse contrast in the interaction with ionizing foil the initial pulse contrast should be high enough not to allow the prepulse trigger the foil ionization-induced transition to opacity. 


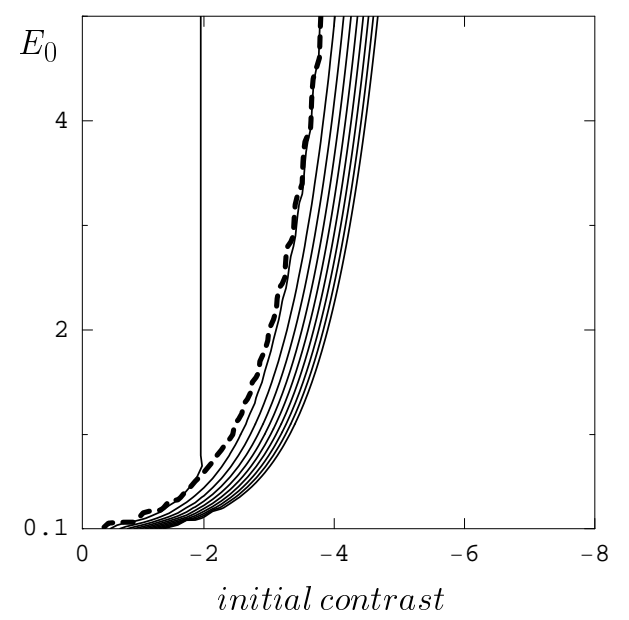

FIG. 3: Dependence of the reflected pulse contrast (the ratio of the pedestal intensity to the main pulse intensity) on the initial pulse contrast (horizontal axis, logarithmic scale) and main pulse dimensionless amplitude (vertical axis, the field is measured in units of $m c \omega / e)$. The contours represent the values $10^{-2 k}, k=1 . .10$. The dashed curve depicts values where the resulting contrast is equal to the initial one.To the right of this curve lie the values of the improved contrast.

\section{HIGH ORDER HARMONIC GENERATION BY THE LINEARLY POLARIZED PULSE.}

Since a circularly polarized pulse does not generate high order harmonics when it ionizes the foil, we consider the ionization of a foil by a linearly polarized pulse. In this case all the formulae of sections 2 and 3 are valid, the only thing that should be taken into account is that in this case the electrons emerge from under their barriers with a momentum distribution that has its maximum at zero. However this distribution is very narrow [16], its width is much less than the typical momentum that an electron acquires in the electric field. Due to this fact the initial momentum distribution can be approximated by Dirac delta function in the expression for the source term. In this case the equation (19) should be modified, and it becomes

$$
\eta=\eta_{0}-\epsilon_{p} \int_{0}^{t-|x| / c} \dot{n}\left(t^{\prime}\right)\left[a(t)-a\left(t^{\prime}\right)\right] d t^{\prime}-\epsilon_{p} \frac{I}{m c^{2}} \frac{\dot{n}(t)}{\eta} .
$$

In what follows we present the results of numerical solution of equation (33) for the same initial pulses as in the preceding section. In Figs. 4 and 5 the form of the reflected and transmitted pulses, plasma density evolution and the spectrum of the reflected pulses are shown for the initial pulse shapes (31) and (32) with the same amplitude and duration as in Figs. 1 and 2. The behavior of the reflected and transmitted pulses is almost the same as in the case of the circularly polarized initial pulse as can be seen from Figs. 4a and 5a. However the evolution of the plasma density is slightly different. In order to illustrate this we present Figs. $4 \mathrm{~b}$ and $5 \mathrm{~b}$, where a step-like function is shown, its behavior is similar to that in the case of circular polarization apart from the step-like feature. This property of the density evolution is due to the ionization. Since the ionization rate is determined by the absolute value of the electric field, in the case of the linear polarization it has two maxima per period of the light wave. The step-like function just demonstrates that the ionization occurs when the electric field amplitude reaches its maximum, while there is almost no ionization in between.

In Figs. 4c and 5c the spectra of the reflected pulses are presented. We see that due to the nonlinear dependence of the ionization rate on the absolute value of the field harmonic generation occurs. Since we consider only normal incidence of the laser pulse on the foil the electric field is parallel to the foil, and that is why only odd harmonics are generated as it can be seen from Figs. 4c and 5c. In order to compare the cases of linear and circular polarizations we also present in Figs. 4c and $5 \mathrm{c}$ the spectra of circularly polarized reflected pulses, that have the same energy as the linearly polarized ones. Here we can clearly see that there is no high order harmonic generation in the case of circularly polarized pulses. Whereas the modification of the spectrum that is due to the ionization blueshift, that was mentioned in Section IV, manifests itself in both cases. However this effect is weak when the plasma mirror works in the condition close to the optimal regime as the transparency-opacity transition is abrupt, since the interaction length is very small, of the order of the skin depth.

Let us estimate the collective efficiency of high order harmonic generation. In order to do so we define the parameter 

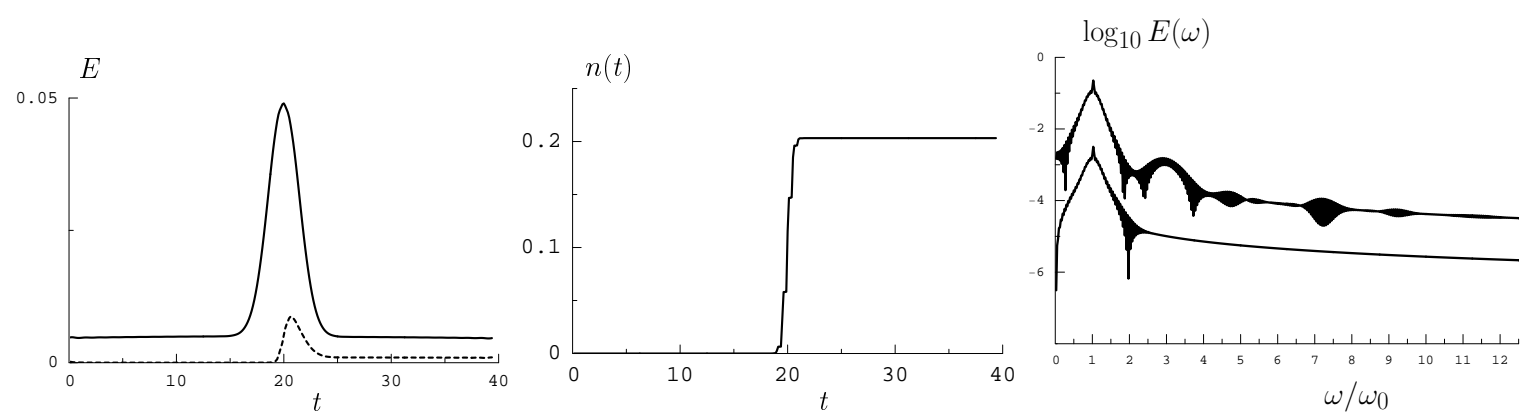

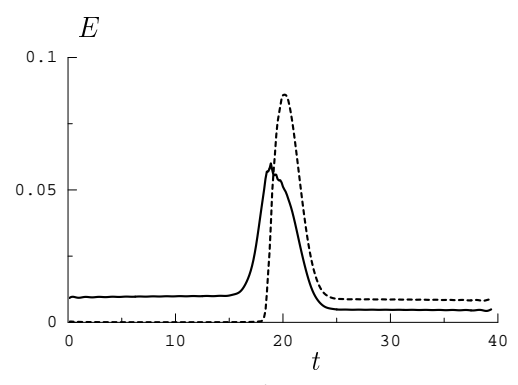

a)

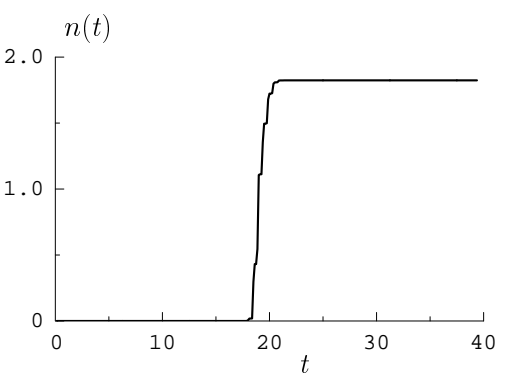

b)

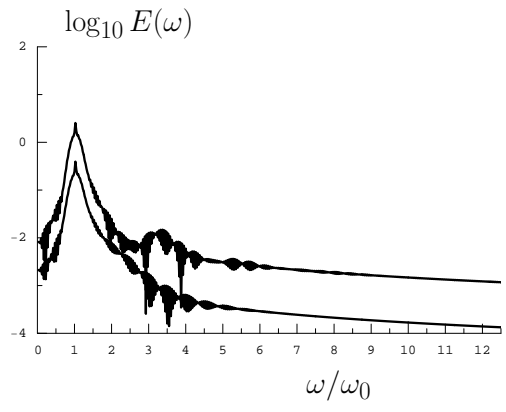

c)

FIG. 4: Evolution of reflected (dotted curve) and transmitted electric field envelopes (a) and plasma density (b) in the case of linear polarization, the spectra of the reflected pulses in the case of linear (upper curve) and circular (lower curve) polarizations for the same pulse energy (c) for initial form of the pulse 31). Time is measured in units of wave period $(2 \pi / \omega)$, field is measured in units of $m c \omega / e$ and density is measured in units of critical density, $n_{c r}$. The parameter $\epsilon_{p} K_{0}=4 \times 10^{3}$. The first row corresponds to the initial pulse amplitude equal to $\eta_{0}^{\max }=0.05$, the second row to $\eta_{0}^{\max }=0.1$.

$R$ :

$$
R=\frac{\int_{\omega=2}^{\omega=\infty} E_{r e f}^{2}(\omega) d \omega}{\int_{\omega=0}^{\omega=\infty} E_{i n}^{2}(\omega) d \omega} .
$$

This parameter has the meaning of the energy that is contained in higher harmonics in the units of initial pulse energy. In other words it measures the efficiency of initial pulse energy transformation into high frequency radiation.

The dependence of $R$ on the initial pulse amplitude is shown in Fig. 6 for different forms of the initial pulse: a) gaussian pulse with pedestal (31) and b) pulse with sine squared envelope (32). The behavior of the curves is almost the same, apart from the fact that in Fig. 6a the parameter $R$ is measured in the units of $10^{-3}$, while in Fig. 6b in the units of $10^{-6}$. For small values of the initial pulse electric field amplitude $R$ is equal to zero. This corresponds to the fact that there is no ionization for low values of $E_{0}$. Then at $E_{0} \approx 0.1$ there is a maximum of $R$. At this value of the initial field amplitude the reflected and transmitted pulses are roughly of the same magnitude. So the high harmonic generation due to foil ionization is most effective where the duration of ionization is the longest.

\section{CONCLUSIONS}

A model for the interaction of a short laser pulse with a thin foil target undergoing field ionization has been derived. The ionization dynamics have been described starting from a kinetic equation for the electrons where the source term includes the ionization rate and the momentum distribution of freed electrons. Energy conservation has been included via a polarization current term. Using the electromagnetic boundary conditions at the foil surface a set of ordinary differential equations has been derived. Such a compact model can be used for simple analysis and preliminary parametric studies of the "plasma mirror" properties of the thin foil, such as prepulse suppression and ultrafast optical shuttering. As an example the transmission, reflection and shaping of a short laser pulse with a pedestal have been studied as a function of the initial pulse contrast and peak intensity. The generation of high harmonics by ionization has also been studied for a linearly polarized pulse. 

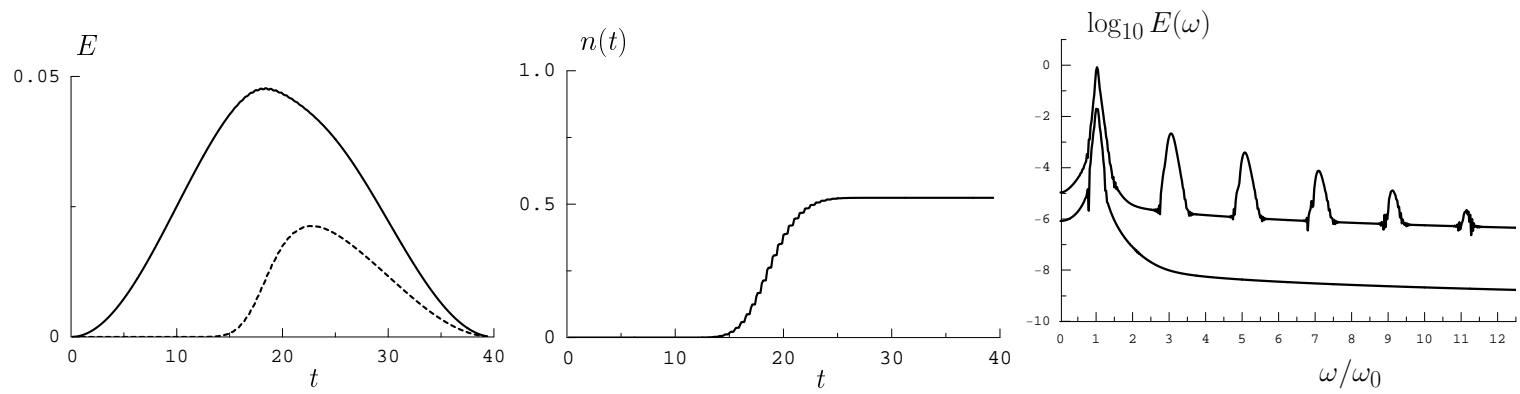

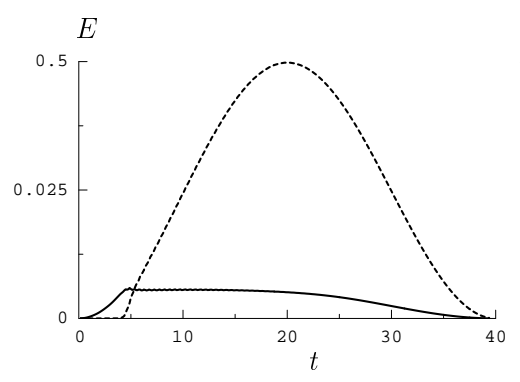

a)

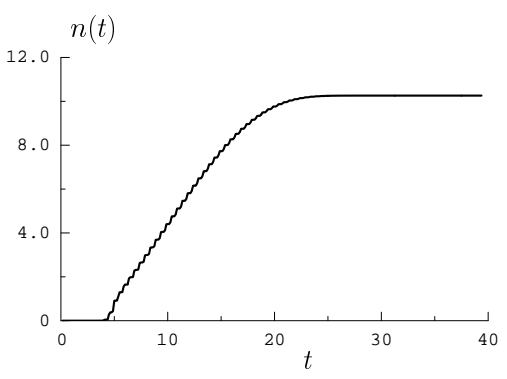

b)

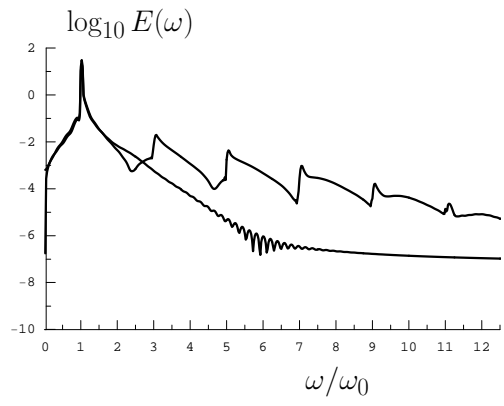

c)

FIG. 5: Evolution of reflected (dotted curve) and transmitted electric field envelopes (a) and plasma density (b) in the case of linear polarization, the spectra of the reflected pulses in the case of linear (upper curve) and circular (lower curve) polarizations for the same pulse energy (c) for the initial form of the pulse (32). Time is measured in units of wave period $(2 \pi / \omega)$, field is measured in units of $m c \omega / e$ and density is measured in units of critical density, $n_{c r}$. The parameter $\epsilon_{p} K_{0}=4 \times 10^{3}$. The first row corresponds to the initial pulse amplitude equal to $\eta_{0}^{\max }=0.05$, the second row to $\eta_{0}^{\max }=0.5$.

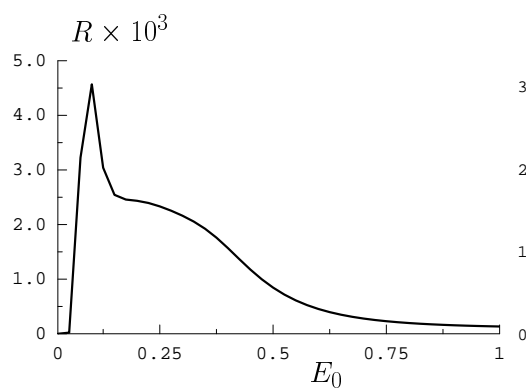

a)

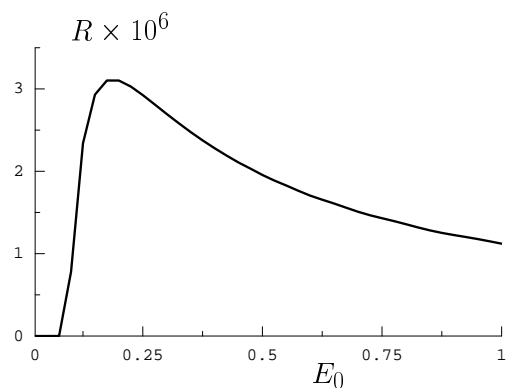

b)

FIG. 6: Effectiveness of harmonic generation vs the initial field amplitude for the cases of initial pulse form (31) (a) and (32) (b). The parameter $\epsilon_{p} K_{0}=4 \times 10^{3}$.

Further extension of this compact model will require the inclusion of multiply ionized species and off-normal incidence laser pulses that can be achieved by the modification of the source term and the proper transformation of the reference frame correspondingly. This will allow the study of the "plasma mirror" operation with respect to the angle of incidence and different states of polarization.

\section{Acknowledgments}

The authors would like to acknowledge fruitful discussions with V. D. Mur, V. S. Popov and V. Yanovsky. This work was supported by the National Science Foundation through the Frontiers in Optical and Coherent Ultrafast Science Center at the University of Michigan and in part by the Russian Fund for Fundamental Research under 
project 04-02-17157.

[1] F. Brunel, J. Opt. Soc. Am. A 7, 521 (1990); M. Lontano, G. Lampis, A. V. Kim, A. M. Sergeev, Physica Scripta T 63, 141 (1996); M. Geissler, G. Tempea, A. Scrinzi, M. Schnuerer, F. Krausz, and T. Brabec, Phys. Rev. Lett. 83, 2930 (1999); A. Bendib, A. Tahraoui, K. Kalache, P. Chessa, and P. Mora, Opt. Commun. 142, 146 (1997); M. V. Chegotov, J. Phys. D: Appl. Phys. 35, 647 (2002).

[2] E. Conejero-Jarque, V. Malishev, and L. Roso, J. Mod. Opt. 44, 563 (1997).

[3] N. E. Andreev, I. L. Beigman, V. V. Kostin, M. E. Veisman, A. M. Umov, Proc. SPIE 3683, 25 (1998).

[4] P. P. Pashinin, Formation and Control of Optical Wavefronts (Proceedings of the Institute of General Physics of the Academy of Sciences of the USSR, Vol 7, Moscow, Nauka, 1987); D. M. Gold, H. Nathel, P. R. Bolton, W. E. White, and L. D. V. Woerkom, Proc. SPIE 1413, 41 (1991); S. Backus, H. C. Kapteyn, M. M. Murnane, D. M. Gold, H. Nathel, and W. White, Opt. Lett. 18, 134 (1993); C. Ziener, P. S. Foster, E. J. Divall, C. J. Hooker, M. H. R. Hutchinson, A. J. Langley, and D. Neely, et. al., J. Appl. Phys. 93, 768 (2003).

[5] B. Dromey, S. Kar, M. Zepf, and P. Foster, Rev. Sci. Instrum. 75, 645 (2004); G. Doumy, F. Quere, O. Gobert, M. Perdrix, Ph. Martin, P. Audebert, J. C. Gauthier, J.-P. Geindre, and T. Wittmann, et. al., Phys. Rev. E 69, 026402 (2004).

[6] I. Watts, M. Zepf, E. Clark, et al., Central Laser Facility Annual Report, RAL, p. 24 (1999).

[7] C. Thaury, F. Quere, J.-P. Geindre, et. al., Nature Phys. 2, 424 (2007).

[8] M. Borghesi, J. Fuchs, S. V. Bulanov, A. J. MacKinnon, P. Patel, and M. Roth, Fus. Sci. Techn. 49, 412 (2006).

[9] D. Neely, P. Foster, A. Robinson, F. Lindau, O. Lundh, A. Persson, C.-G. Wahlstrom, and P. McKenna, Appl. Phys. Lett. 89, 021502 (2006).

[10] P. Monot, G. Doumy, S. Dobosz, et al., Opt. Lett. 29, 893 (2004).

[11] D. Bauer, R. R. E. Salomaa, P. Mulser, Phys. Rev. E 58, 2436 (1998).

[12] S. V. Bulanov, A. Macchi, F. Pegoraro, Phys. Lett. A 245, 439 (1998).

[13] V. Vshivkov, N. Naumova, F. Pegoraro, and S. V. Bulanov, Phys. Plasmas 5, 2727 (1998).

[14] G. Mourou, T. Tajima and S. V. Bulanov, Rev. Mod. Phys. 78, 1 (2006).

[15] L. V. Keldysh, Sov. Phys. JETP 20, 1307 (1965).

[16] A. M. Perelomov, V. S. Popov, and M. V. Terent'ev, Sov. Phys. JETP 23, 924 (1966).

[17] P. Mulser, F. Cornolti, and D. Bauer, Phys. Plasmas 54466 (1998).

[18] F. Cornolti, A. Macchi, and E. Conejero-Jarque, in Superstrong Fields in Plasmas, M. Lontano, G. Mourou, F. Pegoraro, and E. Sindoni, eds.,(American Institute of Physics, Melville, NY) AIP Conf. Proc. 426, 55 (1998).

[19] S. P. Goreslavski and S. V. Popruzhenko, JETP 83, 661 (1996).

[20] V. D. Mur, S. V. Popruzhenko, and V. S. Popov, JETP, 92, 777 (2001).

[21] S. C. Rae and K. Burnett, Phys. Rev. A 46, 1084 (1992).

[22] S. S. Bulanov, A. M. Fedotov, and F. Pegoraro, Pis'ma v ZhETPh, 80, 865 (2004); S. S. Bulanov, A. M. Fedotov, F. Pegoraro, Phys. Rev. E, 71, 016404 (2005).

[23] A. S. Pirozhkov, S. V. Bulanov, T. Zh. Esirkepov, M. Mori, A. Sagisaka, and H. Daido, Phys. Lett. A., 349, 256 (2006); Phys. Plasmas, 13, 1 (2005).

[24] J. K. Koga, N. Naumova, M. Kando, L. N. Tsintsadze, K. Nakajima, S. V. Bulanov, H. Dewa, H. Kotaki, T. Tajima, Phys. Plasmas 7, 5223 (2000). 